\title{
Interactive comment on "Assessment of an ensemble seasonal streamflow forecasting system for Australia" by James C. Bennett et al.
}

\section{Anonymous Referee \#2}

Received and published: 6 September 2017

Summary: This paper presents and assesses a recently developed ensemble seasonal streamflow forecasting system (FoGSS) as an alternative to the stochastic scenarios currently used by many water agencies to plan their operations in Australia. FoGSS is tested on 63 Australian catchments, of which 21 are ephemeral rivers, and was found to overall provide a skilful, reliable and unbiased alternative to stochastic scenarios. The authors however discuss the limitation of FoGSS for very dry ephemeral catchments. Additionally to these findings, the authors describe three experiments designed to improve FoGSS, especially in very dry catchments: 1) the use of historical rainfall forcings (i.e. ESP forecasts) instead of climate forecasts, 2) the use of a different rainfall-runoff model and 3) the use of a Bayesian prior to encourage the FoGSS error model to return climatology forecasts. The main conclusion from these experiments is

Printer-friendly version

Discussion paper 
that the use of a Bayesian prior offers clear benefits in very dry catchments, with some limitations further discussed by the authors.

General comments: Overall I really enjoyed reading this paper as it is very well written and guides the reader nicely from the introduction to the conclusions. The paper fits very well within this HESS special issue, as it presents a newly developed ensemble seasonal streamflow forecasting system for Australia as an alternative to stochastic scenarios for decision-makers. Furthermore, the paper contains a rich number of relevant experiments aimed at improving ensemble seasonal streamflow forecasting, especially in very dry catchments, with some clear conclusions as to the benefits and limitations of each methods. Additionally, this paper refers to and builds nicely on relevant and previous work in this field.

Specific comments and technical corrections:

-P1, L23: "catchments that experience" instead of "catchments that in experience".

-P2, L19-22: Where available, other references for these statements would be good.

-P3, L18: Remove "and" after "rainfall forecasts".

-P3, L30: It would be good to also mention the other forcing variables of the rainfallrunoff model here, i.e., climatology PE. Is temperature not a forcing of the model?

-2.2 Hydrological error model: I agree with reviewer 1 in that the paper could benefit from condensing this section. This would keep the readers more focused on the three experiments nicely described in section 3 and of central importance to the paper's results and conclusions.

-P4, L5: Maybe briefly explain what "heteroscedastic" means as not every reader might be familiar with it.

-P4, Equation 1: Please mention here what TF stands for.

-P4, Equation 2: This equation does not seem vital to mention here so I suggest to

Printer-friendly version

Discussion paper 
remove it.

-P4, L27: "transformed domain" instead of "transform domain". Same on P5, L16.

-P5, L1: "takes" should be deleted.

-P5, L15: It should probably be "zo is the transformed observed streamflow"; "the" is missing.

Interactive

comment

-P5, L19: How is the error in the original domain at $t-1$ calculated?

-3.1.2 Verification scores: Please mention the range of all the scores later displayed (e.g., a CRPSS of $100 \%$ corresponds to a perfect forecast).

-P7, L17: I think that "Catchments and data" should be section 3.1.3 and not 3.1.1.

-P7, L21: Could you please state briefly which interpolation method was used here.

-P8, L12: "alpine" should have a capital "A".

-4.1 Continent-wide performance of the base FoGSS model: I like the focus on the six case study catchments as it allows looking at the results and their differences into more details. However, and since this section is called "continent-wide performance" I think that it could be very beneficial to this section to quickly describe the overall performance of the forecasts for all 63 catchments prior to looking at the six individual case studies. This could be done simply by adding a boxplot of the FoGSS CRPSS for all lead times and target months combined on Figure 3.

-P10, L10-12: This criteria for FoGSS to be characterised as performing well should be stated before describing any results.

-P10, L12-14: Is the negative skill in the Herbert catchment due to the large catchment memory then?

-P10, L14-15: You mention the positive or neutral skill for the Fitzroy catchment, although there are light orange colours (i.e. slightly negative skill) in the plot for this

Printer-friendly version

Discussion paper 
catchment. Could you rephrase this or define "neutral skill".

-P10, L22-23: It is interesting that forecasts are also not reliable for September in the Eppalock catchment. Why is that?

-P10, L39: Please state for which catchments forecasts are generally neutrally skilful by lead-6, i.e. is it for all catchments?

Interactive

comment

-P11, L1: I think that perennial and ephemeral should be swapped here.

-P11, L17: I am not sure what is meant by "irrespective of forcing" here. Please explain further or rephrase.

-P12, L5: It is not obvious why a Budyko-based structure would remain attractive. Could you please argue this slightly for the reader to understand your plan to improve Wapaba instead of using GR2M despite its obvious benefits over the latter.

-P12, L15: Could you please mention that the smaller the $\sigma \mathrm{d}$ values, the stronger the prior (if this is indeed the case), as it was not obvious to me at first.

-P12, L24: Please explain what is meant by "sensibly" here or choose another adjective, i.e. skilfully, reliably, etc.

-P12, L31: This is questionable for perennial catchments for some experiments.

-P13, L5-8: Wouldn't we expect drier months to be better? This needs explaining if so.

-P13, L10: In the Fitzroy catchment the skill is however diminished for longer lead times for forecasts for JAS.

-P14, L21-22: I strongly agree with your belief in the inclusion of seasonal rainfall forecasts in FoGSS. You can however here make this argument stronger as you showed in the paper that the skill from climate forecasts can accumulate to produce skilful long-range total inflow forecasts (mentioned on P12, L36-37). These forecasts being valuable information for reservoir operations in Australia.

Printer-friendly version

Discussion paper 
-Figure 1: State which rainfall-runoff model is used in the FoGSS system.

-Figure 2: Very nice plots! Adding rainfall on these plots could be a nice and useful addition.

-Figures 3 and 13: In the results you mention that FoGSS performs adequately when CRPSS $\geq 0$. Considering this, wouldn't it make sense to modify the colour bar and split the current +5 to -5 range in two sections: +5 to 0 and 0 to -5 ?

comment

-Figures 3, 5, 6, 8, 10, 13: Change CRPS skill scores to CRPSS in the captions and on the plots.

-Figures 3, 4, 13: It would be good to be reminded on the plots or in the captions which of these case study catchments are perennial vs ephemeral.

-Figures 5 to 12: It is sometimes hard to see the difference between two boxplots that you are comparing in the results. Adding notches on the boxplots could make it easier to see for the reader.

-Figures 7 and 11: Both plots for perennial catchments are hard to see, I would recommend rescaling the $y$-axes.

-Figures 10 and 11: Are the numbers 400.25 the $\sigma \mathrm{d}$ values? This is slightly confusing and might be worth changing in the legend.

Interactive comment on Hydrol. Earth Syst. Sci. Discuss., https://doi.org/10.5194/hess-2017395, 2017. 